\title{
Art et Sciences Humaines au Lycée R.M. Adelaide, Aosta, Italie
}

Paola Florio

\section{(2) OpenEdition}

\section{Journals}

Édition électronique

URL : http://journals.openedition.org/esp/422

DOI : $10.4000 /$ esp.422

ISSN : 2532-0319

Éditeur

Centre d'Information sur l'Éducation Bilingue et Plurilingue

\section{Édition imprimée}

Date de publication : 1 décembre 2014

Pagination : 10-13

ISSN : 1127-266X

\section{Référence électronique}

Paola Florio, « Art et Sciences Humaines au Lycée R.M. Adelaide, Aosta, Italie », Éducation et sociétés plurilingues [En ligne], 37 | 2014, mis en ligne le 01 octobre 2015, consulté le 15 septembre 2020. URL : http://journals.openedition.org/esp/422 


\section{ART ET SCIENCES HUMAINES AU LYGÉE R.M. ADELAIDE, AOSTA, ITALIE}

\section{Paola Florio}

Il progetto "ART et Sciences Humaines" ha attuato un approfondimento delle conoscenze, delle abilità e delle competenze delle discipline coinvolte partendo da moduli teorici che si sono declinati nella possibilità di fare esperienze pratiche sul campo sempre accompagnate da un'attenta osservazione partecipante da parte dell'alunno. Tale progettualità mira a attivare un cambiamento culturale (1), un'apertura mentale, un approccio più approfondito ad una tematica legata al mondo dell'arte tramite $i$ seguenti passaggi attuativi: formazione, visita al Museo di Arte Moderna presso il Castello Gamba, studio e elaborazione di materiali didattici, esperienza pratica di attività di riflessione sull'arte con $i$ bambini della scuola primaria, in lingua veicolare francese, accompagnata da una produzione artistica da parte dei bambini guidata dalle ragazze di IV A del Liceo delle Scienze Umane di Aosta.

Das Projekt "Kunst und Geisteswissenschaften" hat Strategien entwickelt, um das Erlernen der Kunst und gleichzeitig der Zielsprache interessant und motivierend zu gestalten. Die Umsetzung des Projekts geht einher mit einer Vertiefung der Kompetenzen, des Wissens und der Fähigkeiten und Fertigkeiten in den betroffenen Disziplinen. Dies geschieht über Theorie-Module und praktische Erfahrungen. Die Schüler wurden bei ihrer Arbeit aufmerksamen und teilnehmend beobachtet. Dieses Projekt zielt darauf ab, den kulturellen Horizont zu erweitern, ein aufgeschlossenes Verhalten zu fördern und ein gründlicheres Herangehen an ein die Welt der Kunst betreffendes Thema zu ermöglichen. Dieses Ziel soll in vier Etappen umgesetzt werden: Ausbildung, Besuch des Museums für Moderne Kunst im Schloss Gamba, Herstellung und Entwicklung von Unterrichtsmaterialien, praktische Erfahrung in der Reflexion über künstlerische Aktivitäten bei Grundschulkindern, in der Unterrichtssprache Französisch, begleitet von künstlerischen Produktionen der Kinder, die von den Schülerinnen des humanistischen Gymnasiums von Aoste angeleitet werden.

The "Art et Sciences Humaines" project devised strategies to make studying art and the target language interesting and stimulating. It implied improving one's knowledge, competence and expertise in the subjects concerned, based on theoretical modules leading to practical experience. The students were constantly called upon to observe closely and participate actively. The project aims to permit cultural change (2), an open mind, an indepth approach of a subject connected to the world of art thanks to training, visiting the Museum of Modern Art in the Château Gamba, analyzing and developing educational material, and having the practical experience of thinking out artistic activities for primary school children in French. All this was accompanied by the pupils' own artistic production, under the guidance of the girls in section IV-A at the Lycée des Sciences Humaines in Aosta. 
Art et Sciences Humaines au Lycée R.M. Adelaide, Aosta, Italie

P. FLORIO

VERSION FRANÇAISE
L e projet a commencé par une formation "Classes Musée" du Château Gamba de Châtillon au mois d'octobre 2013 sous la supervision de deux Formateurs experts du Musée du Louvre, Collaborateurs de la Direction de la Politique des Publics et de l'éducation artistique.

Puis, il y a eu la visite de la classe 4A UMA, au Château Gamba le 6 Décembre 2013.

Gratuitement, à cette occasion, au Château Gamba de Châtillon, les élèves ont réalisé le laboratoire IMAGES et MOTS. Le XX siècle à travers l'art e la littérature, en italien, français et anglais. L'analyse et la contextualisation des œuvres d'art et la présentation des artistes et des mouvements littéraires ont été développées par la lecture et la discussion des passages de textes littéraires italiens, français et anglais. Cette approche a permis de mieux comprendre le climat historique et culturel du XX siècle. La durée de la visite a été de 2 h.

Grâce à ce travail, la classe a eu l'occasion d'approfondir les disciplines suivantes: langue et littérature italienne, française et anglaise, histoire, philosophie, histoire de l'art, sciences humaines, en particulier la pédagogie, à travers la méthodologie de la recherche-action dans la phase suivante de collaboration avec l'école primaire.

Le but principal était de consolider les connaissances, les aptitudes et les compétences suivantes: capacité d'observer, décrire, construire des analogies et des comparaisons; savoir effectuer un jugement esthétique et comprendre les valeurs historiques et culturelles des œuvres d'art, savoir formuler des hypothèses, se poser des questions et savoir comment interpréter une œuvre d'art face au contexte historique et culturel dans lequel elle a été produite; développer la compréhension et la création de liens entre les différentes formes artistiques et entre différents époques et contextes.

Une autre étape du travail des étudiantes (2) s'est déroulée sur le terrain en visite à Florence, où elles ont pu suivre un parcours de grande importance artistique et culturelle avec le but d'élaborer le programme de la quatrième année du Lycée des Sciences Humaines, en particulier pour ce qui concerne l'anthropologie, la philosophie et l'histoire de l'art.

En particulier, on a voulu utiliser un enseignement vivant en allant sur place à Florence pour voir le Musée d'anthropologie et d'ethnologie (avec visite guidée), le Museo Galileo (avec visite guidée), le complexe de la cathédrale (l'église de Santa Maria del Fiore), la Galerie des Uffizi, le Palazzo Vecchio et les Jardins de Boboli. 
Art et Sciences Humaines au Lycée R.M. Adelaide, Aosta, Italie

P. FLORIO
Ensuite le matériel didactique pour les enfants a été crée, concernant en particulier la Renaissance italienne et l'Impressionnisme français, avec une réflexion, en français, en deux classes cinquièmes de l'école primaire "Saint François" à Aoste.

Après cela, il y a eu deux rencontres, au mois d'avril, d'expérience pratique de réflexion sur l'art avec les enfants de l'école primaire, en français, accompagnée par une production de peintures.

Les ressources humaines des deux écoles impliquées dans le projet étaient: le professeur Filippo Giannone, expert en histoire de l'art, Mme. Alessandra Joly, professeur de français, Mme. Annamaria Istria, professeur d'anglais, Mme. Florio Paola, professeur de Philosophie et Sciences Humaines et les enseignantes Frassy Sandra, Petretto Mariella, Godioz Ornella, Elena Roncari.

Les élèves, dans l'évaluation finale du projet, ont exprimé leur satisfaction d'avoir pu enrichir leur culture, d'avoir appris à raisonner de façon plus analytique sur les idées des artistes et de comprendre avec plus de profondeur leurs pensées.

Même la préparation initiale à l'intérieur de la classe, pendant les heures de français et d'anglais, en analysant des textes littéraires conceptuellement liés aux peintures d'art moderne du Château de Gamba, a été une expérience très enrichissante.

Ensuite la visite à Florence, en mars 2014, a suscité beaucoup d'émotions, toutes positives, certainement inoubliables: les étudiantes ont pu voir les œuvres des plus grands artistes italiens. Les Uffizi ont été définis par les étudiantes comme "un musée extraordinaire, avec un grand nombre d'œuvres impressionnantes et magnifiques". Certaines filles ont avoué "ne pas être au courant de la beauté de Florence jusqu'au moment où elles se sont trouvées immergées dans sa beauté" et elles ont exprimé le désir de vouloir y retourner. Le voyage à Florence, décrit comme "fantastique et très intéressant», a renforcé la cohésion de la classe et a déclenché la curiosité à propos de ce qu'on observe, en développant un regard critique.

L'expérience avec les enfants a été jugé intéressante et instructive, même pour l'orientation professionnelle. En outre, elle s'est révélée très profonde, pour leur propre identité, permettant de se sentir en mesure d'expliquer, d'interagir, de collaborer avec des élèves plus jeunes en offrant une expérience précieuse de peer education. Plusieurs étudiantes ont dit qu'elles ont obtenu la confirmation que "travailler avec les enfants c'est le travail que je veux pour moi", et elles ont pu choisir clairement leurs études universitaires futures. D'autres filles au contraire ont dit avoir beaucoup de préoccupation de travailler avec les enfants à l'école, parce qu'elles 
Art et Sciences Humaines au Lycée R.M. Adelaide, Aosta, Italie

P. FLORIO

\section{VERSION ITALIENNE}

avaient peur de "ne pas avoir leur attention ou de ne pas être capables de bien communiquer avec eux". Pourtant le climat psychologique était fait d'harmonie et d'amusement pour les enfants, mais aussi de concentration. Une jeune fille a dit "je n'ai pas seulement appris quelque chose, mais j'ai aussi pu faire l'expérience merveilleuse de la sensation d'enseigner, d'être capable d'engager les autres et de leur faire naître de l'intérêt pour l'art."

Certaines étudiantes ont remarqué que l'aspect le plus important a été d'observer des œuvres personnellement, en retrouvant des concepts qu'elles ont étudiés tout au long de l'année sur les livres, ainsi elles ont pu comprendre des détails en profondeur que, autrement, elles n'auraient pas été capables de voir. Par exemple, Galileo Galilei a été étudié en italien, en philosophie et en physique, et le fait de pouvoir visiter le Musée Galileo, surtout avec tous les instruments originaux, pour elles a été le plus intéressant. Une étudiante a dit: "Je suis vraiment satisfaite de ce que j'ai vécu."

Personnellement, je pense que ceci est le but le plus élevé que nous pouvons demander à l'école d'aujourd'hui: travailler en regardant "grandir, mûrir, évoluer" les élèves dans un esprit de satisfaction avec ce qu'ils font.

Il progetto è iniziato con la formazione della docente Florio Paola mediante la partecipazione allo Stage de formation "Classes Musée" al Castello Gamba de Chatillon il 15.10.13 sotto la supervisione di due Experts formateurs du Musée du Louvre collaborateurs de la Direction de la politique des publics et de l'éducation artistique.

Successivamente si è svolta la visita, da parte della classe 4A UMA, al Castello Gamba il giorno 6 dicembre 2013.

Gratuitamente, in tale occasione, presso il Castello Gamba di Chatillon, le alunne hanno svolto il laboratorio IMMAGINI e PAROLE. Il XX secolo attraverso l'arte e la letteratura. In lingua italiana, francese e inglese. L'analisi e la contestualizzazione delle opere d'arte e la presentazione di artisti e movimenti, viene intervallata dalla lettura e il commento di brani di testi di letteratura italiana, francese e inglese per meglio cogliere il clima storico-culturale del periodo e per offrire ulteriori spunti di riflessione. La durata della visita è stata di $2 \mathrm{~h}$.

Grazie a questo lavoro la classe ha avuto modo di approfondire le seguenti discipline: lingua e letteratura italiana, francese ed inglese, storia, filosofia, storia dell'arte e scienze umane ed in particolare pedagogia con metodologia di ricerca-azione nella fase succes- 
Art et Sciences Humaines au Lycée R.M. Adelaide, Aosta, Italie

P. FLORIO siva di collaborazione con la scuola primaria.

La finalità principale è stata di consolidare le seguenti conoscenze, capacità e competenze: saper osservare, descrivere, costruire analogie e confronti, attribuire un giudizio estetico e valori storico-culturali alle opere d'arte, formulare ipotesi e porre problemi e saper interpretare un'opera d'arte alla luce del contesto storicoculturale in cui è stata prodotta, comprendendo e creando collegamenti fra le diverse espressioni artistiche e fra epoche e contesti differenti.

Come ulteriore momento di approfondimento sul campo le studentesse sono andate in visita didattica a Firenze, dove hanno potuto seguire un percorso artistico-culturale di grande importanza con la finalità di approfondire i programmi della quarta del liceo delle scienze umane soprattutto in antropologia, filosofia e storia dell'arte.

In particolare si è inteso quindi utilizzare una didattica viva andando sul campo ad ammirare: il Museo Antropologico ed Etnologico (con visita guidata), il Museo Galileo (con visita guidata), il complesso del Duomo, la Chiesa di Santa Maria del Fiore, la Galleria degli Uffizi, il Palazzo Vecchio ed i Giardini di Boboli. In seguito vi è stata l'elaborazione di materiali didattici di campo artistico in particolare il Rinascimento italiano e l'Impressionismo francese per una riflessione, in lingua francese, in due classi quinte della scuola primaria.

Una volta pronti i materiali didattici sono state svolte due mattinate, ad aprile, di esperienza pratica, di attività di riflessione sull'arte con i bambini della primaria, in lingua francese, accompagnata da una produzione artistica di tipo pittorico, da parte dei bambini, guidata dalle ragazze della IV A UMA del Liceo R.M.Adelaide di Aosta (1).

Le classi coinvolte sono quindi state, oltre alla classe IV A UMA, due classi quinte dell'Istituzione Scolastica "San Francesco" di Aosta. Le risorse umane delle due scuole implicate nel progetto sono state: il Prof. Giannone Filippo, esperto di Storia dell'Arte, la Prof.ssa Joly Alessandra, docente di Francese, la Prof.ssa Istria Annamaria, docente di Inglese, la Prof.ssa Florio Paola, docente di Scienze Umane e Filosofia e le docenti di classe quinta primaria Frassy Sandra, Petretto Mariella, Godioz Ornella, Elena Roncari.

Le studentesse, nella valutazione conclusiva del progetto, hanno espresso la loro soddisfazione per essere riuscite ad arricchire il loro bagaglio culturale, per essere riuscite a ragionare in modo più analitico sulle idee degli artisti nel realizzare le loro opere e di 
Art et Sciences Humaines au Lycée R.M. Adelaide, Aosta, Italie

P. FLORIO capire con più profondità i loro pensieri.

Anche solo l'iniziale preparazione in classe nelle ore di lingua francese e inglese, analizzando testi letterari collegati concettualmente con i quadri di arte moderna del Castello Gamba, è stato un momento molto arricchente.

In seguito la visita a Firenze, a marzo 2014, ha suscitato molte emozioni, tutte positive, sicuramente indimenticabili per aver potuto vedere le opere dei più grandi artisti italiani. Gli Uffizi sono stati da loro definiti come "una struttura museale straordinaria con un numero esorbitante di opere imponenti e magnifiche". Alcune ragazze hanno ammesso di "non essere state consapevoli della bellezza di Firenze fino al momento in cui non si sono trovate immerse nella sua Bellezza" ed hanno espresso il desiderio di volerci tornare. Il viaggio a Firenze, definito "fantastico e interessantissimo", ha unito maggiormente il gruppo classe ed ha fatto nascere curiosità verso ciò che si osserva, ponendosi davanti ad un'opera d'arte o culturale con occhio critico.

L'esperienza sul campo con i bambini è stata giudicata interessante e istruttiva, anche in vista di un orientamento per il proprio futuro professionale. Inoltre è stata vissuta in modo molto profondo, lasciando molti ricordi di accrescimento del proprio sé, del sentirsi capace di spiegare, interagire, collaborare con studenti più piccoli realizzando una valida esperienza di peer education. Diverse studentesse hanno affermato di aver avuto la conferma che "lavorare con $i$ bambini è proprio il lavoro che voglio per me", non solo, ma sono riuscite così ad avere chiara la facoltà Universitaria da scegliere l'anno prossimo, talune in ambito pedagogico altre in ambito psicologico. Altre hanno confessato che inizialmente erano abbastanza spaventate di dover seguire i bambini a scuola poiché avevano paura di "non avere la loro attenzione, di annoiarli o di non riuscire a comunicare bene con loro". Il clima psicologico che poi si è creato con i bambini è stato di armonia e divertimento, con anche però molto impegno da parte dei piccoli. Una ragazza ha detto "di non aver solo imparato, ma di aver anche potuto provare la bellissima sensazione di trasmettere qualche insegnamento agli altri, riuscendo a coinvolgerli e a farli interessare all'Arte".

Alcune studentesse hanno sottolineato come l'aspetto più importante sia stato poter osservare dal vivo le opere, i concetti e le strutture che hanno studiato tutto l'anno sui libri, riuscendo così a cogliere in profondità particolari che altrimenti non sarebbero riuscite a vedere. Ad esempio Galileo Galilei era stato studiato in Italiano, Filosofia e Fisica e poter vedere il Museo Galileo, con anche tutti gli strumenti originali, è stato per loro più interessante 
Art et Sciences Humaines au Lycée R.M. Adelaide, Aosta, Italie

P. FLORIO e coinvolgente.

Un'alunna è arrivata a dire"sono veramente soddisfatta di ciò che ho fatto".

Personalmente penso che questo sia il più alto obiettivo che si possa porre la scuola oggi: lavorare vedendo "crescere, maturare, evolvere" i propri studenti con spirito di soddisfazione.

\section{NOTE}

(1) Viser un changement de culture (cambiamento culturale) signifie apprendre à étudier de façon plus interdisciplinaire. C'est aussi bien sûr un enrichissement culturel par l'ouverture vers l'Autre, vers la littérature, la peinture, les enfants, le futur, la responsabilité et la liberté de rêver ce qu'on veut faire dans la vie. Il s'agit aussi d'un changement INTERculturel, la culture d'origine change pendant que le temps passe, les racines d'origine restent, mais l'arbre (les filles) changent lentement leur approche au monde, au passé, au quotidien...

(2) L'âge des élèves de la classe IV A est de 18 ans. En Italie, le BAC est à 19 ans, à la fin de la classe V. Le lycée est mixte, mais cette classe est uniquement composée de jeunes filles. 